\title{
PILONIDAL SINUS
}

\author{
D J Jones
}

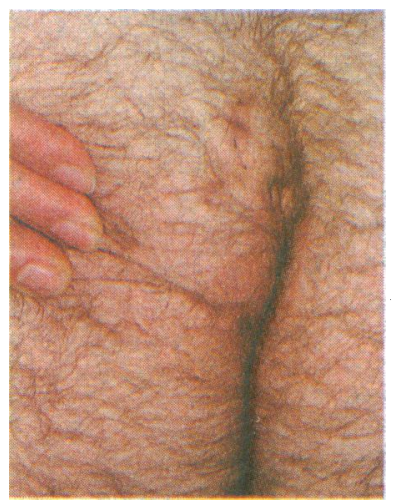

Pilonidal sinus with hairy natal cleft and secondary lateral openings.

\section{What is a pilonidal sinus?}

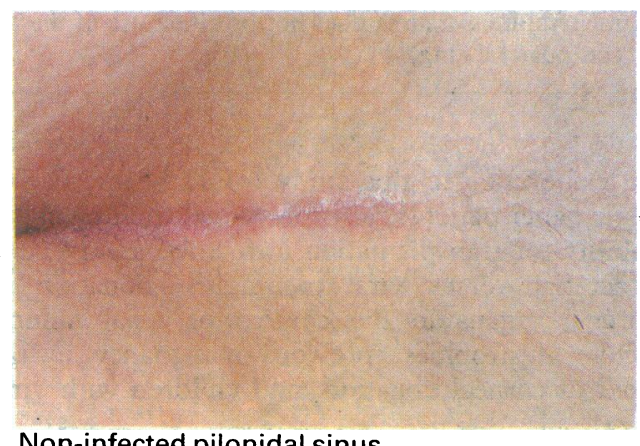

Pilonidal disease is a common affliction of young adults that causes considerable suffering, inconvenience, and time lost from work. Treatment varies greatly and is often less than satisfactory. The rate of recurrence after surgery is purported to be as high as $50 \%$.

A pilonidal sinus consists of a characteristic midline opening or series of openings in the natal cleft about $5 \mathrm{~cm}$ from the anus. The skin enters the sinus, giving the opening a smooth edge. This primary track leads into a subcutaneous cavity which contains granulation tissue and usually a nest of hairs, which may be seen projecting through the skin opening. Many patients have secondary lateral openings $2-5 \mathrm{~cm}$ above the midline pit. The skin opening and superficial portion of the track are lined with squamous cell epithelium, but the deep cavity and its extensions are not.

\section{Pathogenesis}

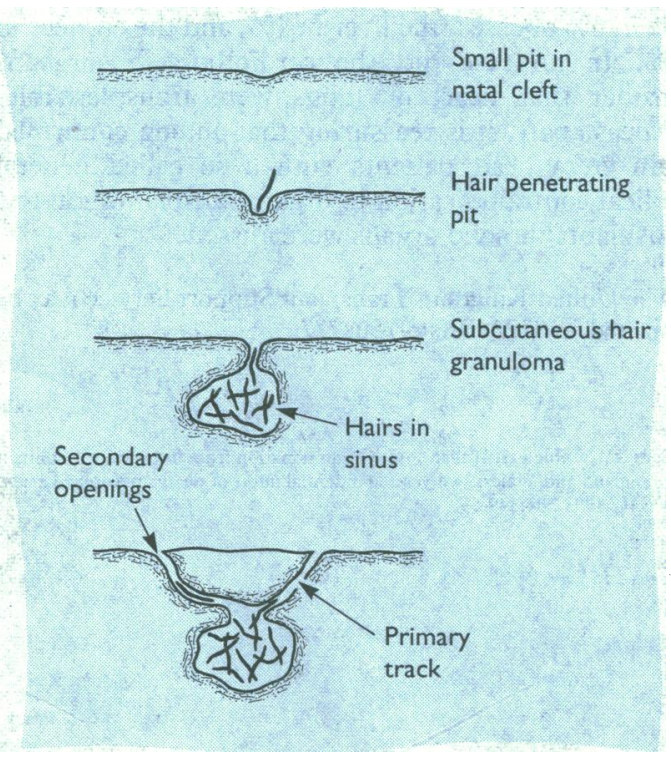

Mechanism of pathogenesis of a pilonidal sinus.
Pilonidal sinuses are now widely accepted to be acquired abnormalities, although theories of a congenital origin, from postcoccygeal cells or vestigial scent glands, were once popular. Sinuses to the neural canal and dura are rare; they usually present in childhood and are in the lumbar rather than sacral region.

Pilonidal disease starts at the onset of puberty, when sex hormones start acting on pilosebaceous glands in the natal cleft. A hair follicle becomes distended with keratin and subsequently infected, leading to a folliculitis and an abscess which extends down into the subcutaneous fat. Tracks spread out of the cavity in the direction of the neighbouring hair growth, which is towards the patient's head in over $90 \%$ of cases. A small proportion have tracks which pass caudally, and these pilonidal sinuses tend to be closer to the anus. Hairs are drilled or sucked into the cavity owing to friction with movement of the buttocks. Barbs on the hairs prevent their expulsion, so they become trapped, provoking a foreign body type reaction and infection. 


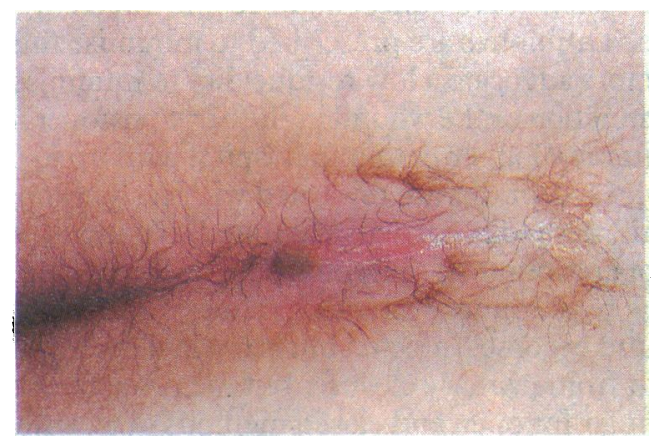

Pilonidal sinus with discharge.
Pilonidal sinuses present from the age of puberty to about 40 years of age, but rarely in older people. Three quarters of patients are men, predominantly in their 20 s. About $1 \%$ of men in the age group at risk develop a pilonidal sinus. Affected women tend to be younger, which is consistent with their earlier onset of puberty. Patients are usually, but not invariably, dark and hairy, and are often obese. Pilonidal sinues are less common among Africans and Asians owing to different hair characteristics and growth patterns.

Half of the affected patients present as emergencies with an acute pilonidal abscess; the remainder have fluctuating discomfort and chronic infection with a foul smelling discharge. Examination reveals the characteristic opening in the natal cleft, through which a tuft of hair may be seen emerging.

\section{Treatment}

Meticulous hygiene and shaving are important for all forms of treatment. Shaving may be stopped once the wound has healed

\section{Management of open granulating wounds}

- Adequate drainage combined with the prevention of pocketing and bridging are more important than the type of dressing used

- Tight packing is contraindicated as it prevents wound drainage and causes discomfort

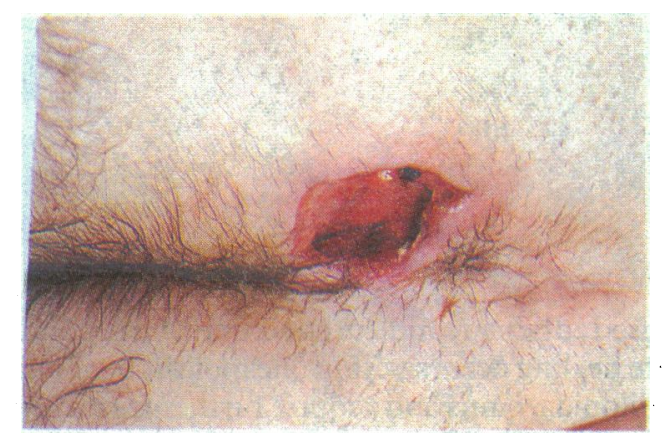

Pilonidal sinus which has been excised and left open to heal by secondary intention.

\section{Principal surgical options}

Non-infected sinus-Excision and primary closure or

Excision and laying open

Infected sinus or abscess-Drainage and excision

Healing by secondary intention
}

Many different treatments have been advocated with conflicting enthusiasm, suggesting that none are perfect. Some patients can be treated on an outpatient or day case basis while others require periods in hospital. A balance has to be struck between minimising inpatient treatment without compromising lasting healing.

\section{Curettage}

If the sinus is small and not infected hairs can be removed by using forceps or small bristle brushes and the surrounding skin shaved weekly. In the absence of a persisting foreign body reaction to the hair healing may occur.

\section{Phenol injection}

Before injection of phenol the surrounding skin is shaved and protected with petroleum jelly. Phenol is injected down the opening of the main tract, left for one minute, and then expressed. The procedure is repeated two more times, giving a total exposure to phenol of three minutes. A light dressing is applied, and healing takes between three and six weeks. Phenol injection may be combined with local excision of the sinus.

Simple curettage and phenol injection avoid hospital admission but require meticulous and prolonged attention to the sinus if recurrence is to be avoided.

\section{Excision}

Excision of pits - Midline pits and lateral openings can be excised with a small area (less than $0.5 \mathrm{~cm}$ ) of surrounding skin under local anaesthesia and the cavity curetted to remove the hair granuloma. The sinus requires frequent changes of dressings and weekly brushing with a small bristle brush. Healing occurs in about six weeks.

Laying open - If the acquired theory of aetiology is accepted it is not necessary to perform an excision down to the presacral fascia. Laying the sinus open to permit adequate drainage allows healing by secondary intention. Frequent changes of dressings and close supervision are necessary to prevent pocketing. Regular rubbing with a finger avoids premature closure or bridging of the skin edges over an incompletely healed cavity; these tend to occur as healing nears completion, when attention to the wound is waning. 
aid open pilonidal sinus with a dressing of Silastic foam.

Excision with primary suture - The pilonidal sinus is excised and the wound sutured under general anaesthesia by using deep tension sutures tied over a gauze dressing. This approximates the skin edges and occludes the dead space where blood and serum could collect and become infected. Broad spectrum prophylactic antibiotics are prescribed to minimise the risks of infection. Many doctors advocate a low residue diet to minimise defecation and faecal contamination of the wound in the early postoperative period. Primary closure requires a longer period in hospital with up to one week of bed rest. Patients who accept this are rewarded with early complete healing after two weeks. This method is particularly suited to patients who find it difficult to accept an open wound. If the wound becomes infected it is reopened and allowed to heal by secondary intention. However, delayed healing may occur if the wound becomes infected but is not laid open. Many patients undoubtedly benefit from primary closure, but the proportions healed at two months are similar for open and closed methods of treatment.

\section{Comparison of primary closure and laying open}

Primary closure
Quicker healing
Success depends on the surgeon
Early return to work
Failure delays healing
Longer period in hospital
Minimal wound care

Laying open

Slower healing

Effective in most hands

Open wound may delay return to work

Shorter period in hospital

Active wound care with frequent wound dressings

The proportions healed at two months are similar for both forms of treatment
Excision with primary suture and flattening of the natal cleft-Techniques which flatten the natal cleft help to diminish the accumulation of hair and reduce friction with movement. The sinus is excised asymmetrically, with one flap being undermined and overlapped over the opposite flap. Alternatively a myocutaneous flap or Z-plasty flap can be used to close the wound. These techniques are more demanding and require a period in hospital. If the operation is unsuccessful patients endure a more prolonged period of healing owing to the size and complexity of the wound.

\section{Causes of recurrence}

\section{Causes of recurrence}

- Neglect of wound care

- Persisting poorly drained tracks

- Recurrent infection of hair follicles

- Midline scars
Early recurrence is usually due to the persistence of tracks lined with granulation tissue in an incompletely healed wound. Late recurrence is caused by repeated infection of hair follicles. Procedures which leave a midline scar are most susceptible to further hair penetration. Healing by secondary intention leaves a flat, broad, hairless scar which reduces buttock friction and is less susceptible to penetration by hair.

\section{Pilonidal abscess}

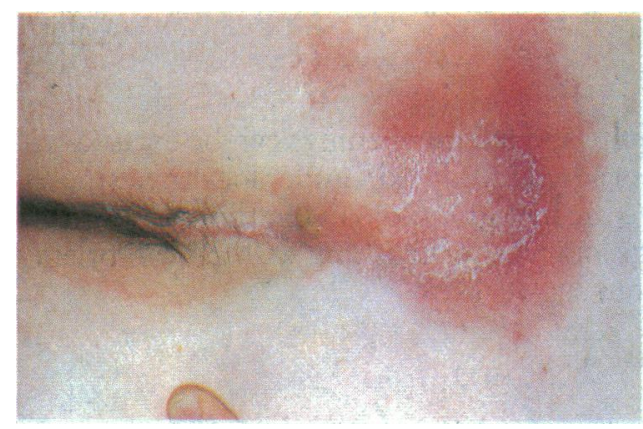

Pilonidal abscess.
A pilonidal abscess is incised, drained, and curetted free of hairs and granulation tissue. Complete healing occurs within one month, but almost a third of patients develop a pilonidal sinus and require further treatment.

Recurrence is reduced if sinus pits are recognised and excised at the time of primary drainage, although these may be difficult to recognise in the presence of infection and oedema. The pit is more easily distinguished and excised a week after primary drainage, when the oedema has resolved. This reduces the patient's chance of developing a chronic sinus and avoids readmission.

The photographs were produced by the department of medical illustration, Salford Health Authority.

$\mathrm{Mr} \mathrm{D} J$ Jones is lecturer and honarary senior registrar in general surgery, Hope Hospital, Salford.

The ABC of Colorectal Diseases has been edited by Mr D J Jones and Professor M H Irving, department of surgery, Hope Hospital, Salford. 